\section{Vaccine-autism link}

\section{discounted, but effect of}

\section{"study" is unknown}

A $\mathrm{s}$ the authors of a controversial study that linked the measles, mumps and rubella (MMR) vaccine and autism fend off allegations of professional misconduct, debate over the study's impact on immunization rates continues to rage in Canada.

The hard evidence, though, remains elusive as officials say Canada's tracking system is too woefully inadequate to accurately ascertain the controversy's effect in Canada.

Many immunologists and public health experts believe the study triggered a worldwide decline in the number of children receiving the vaccine (Lancet 1998;35I:634-4I, removed online). In the United Kingdom, coverage rates for the vaccine dropped to $75 \%$.

There are little empirical data of a similar decline in Canada. The Public Health Agency of Canada (PHAC) reported in the 2006 National Immunization Survey that approximately 9r\% of Canadian children had received the MMR vaccine. That rate was only

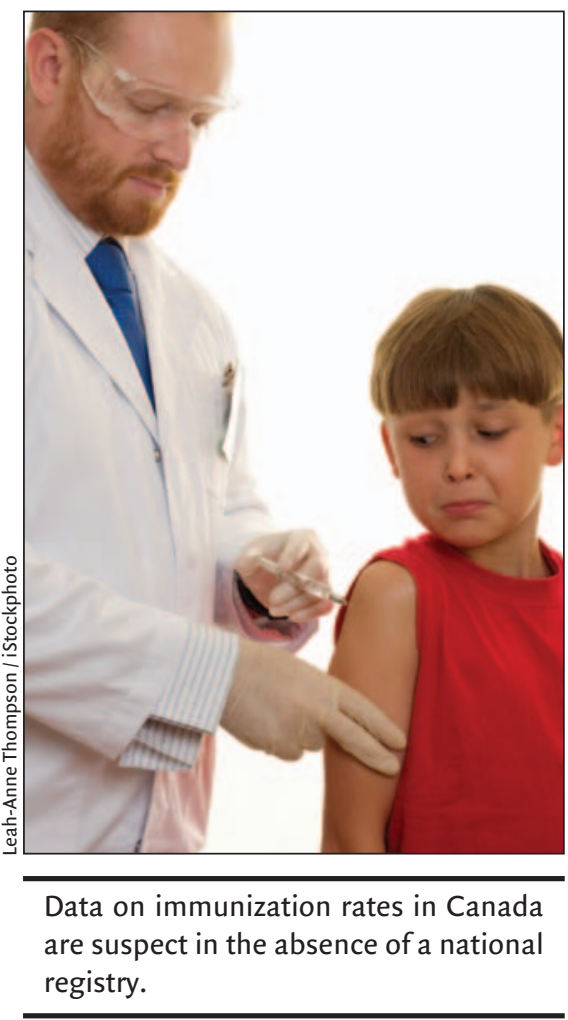

slightly lower than the $94 \%$ coverage projected in 1997.

That suggests that coverage rates for the vaccine haven't been impacted and that parents haven't stopped doctors from administering the vaccine, says Mahnaz Farhang Mehr, chief of immunization programs for PHAC. "But it probably ... affected parent's confidence in the vaccine."

Public concern about an MMRautism link resurfaced this summer after the UK General Medical Council announced it would hold hearings into allegations of professional misconduct against Dr. Andrew Wakefield, Professor John Walker-Smith and Professor Simon Murch, 3 of the original authors of the Lancet study. Subsequent clinical studies have since failed to confirm the findings. In 2004, Io of the 12 experts who collaborated with Wakefield retracted the study.

Now before the UK General Medical Council are such alleged ethical breaches as paying children at birthday parties to provide blood samples, and failing to inform the Lancet that Wakefield was advising lawyers who were representing parents who believed their children were adversely affected by the vaccine.

Although the study's findings have been widely discredited, immunologists and public health officials remain concerned.

"Right now, nobody is able to document or trace the true impact of the MMR study," says Mary Appleton, the senior manager for the Canadian Coalition for Immunization Awareness and Promotion. "Our registration system is simply insufficient."

In Canada and the United States, the health care systems get data on immunization coverage rates from a variety of sources including patient records. However, national surveys in each country rely most heavily on public opinion survey methodology to come up with coverage rate estimates, an approach that many in the field believe is little more than an educated guess.

Among Canadian provinces, only Manitoba has a formal system in place that requires physicians to record in a province-wide registry every immunization administered, Fahrang Mehr said. Without a national registry, it is impos- sible to determine with absolute certainty what kind of "uptake" there has been on all the major childhood vaccines (CMAJ 2007:176[13]:I8II-3).

A widely cited 2006 study found that less than $70 \%$ of Canadian 2-year-olds received their full range of vaccines (Pediatrics 2006;117:595-602). The result was especially troubling given the fact these children had, on average, visited a physician Ig times.

Dr. Joanne Embree, the head of pediatric infectious diseases at the University of Manitoba, says there is widespread concern the lack of specific data, and poor vaccine coverage, is a by-product of the difficulty many Canadians have in finding a regular family doctor or pediatrician.

Embree says parents who rely heavily on walk-in clinics for their health care needs often slip through the cracks of the system. They only approach a physician when their children have a specific health problem, and the subject of whether vaccines are up to date never arises, she adds. "If you do not have a family doctor then you likely don't have someone to remind you to get your vaccines on time."

The shortage of family doctors is also depriving the health care system of much-needed data on how many parents refuse to allow their children to be vaccinated, Embree adds. Almost all family physicians and pediatricians can anecdotally reference families that decline certain vaccines. - Dan Lett, Winnipeg

DOI:I0.1503/cmaj.07II99

\section{US Medicare will stop paying}

\section{for preventable errors}

I n a major policy change projected to save lives and millions of dollars, Medicare will stop paying US hospitals to correct 8 preventable medical errors caused by their own negligence, commencing in October 2008.

The new payment guidelines are part of a 3-year effort by Medicare, the largest health care payer in the United States, to improve care for elderly and 
disabled Americans. Under guidelines issued in August, Medicare will no longer pay hospitals to retrieve objects, such as sponges or surgical tools, left in patients after surgery, or to treat problems arising from air embolisms or incompatible blood transfusions. Nor will it pay the additional costs of treating bedsores developed while in the hospital, injuries caused by hospital falls, or infections arising from prolonged use of urinary and vascular catheters or after coronary artery bypass surgery.

"The overall mission of the Medicare program is changing," said Herb Kuhn, deputy administrator of the Centers for Medicare \& Medicaid Services. "Our efforts are focused on becoming an active purchaser, rather than a passive payer, of health care."

The Bush administration estimates the new regulations will save $\$ 20$ million annually, but others predict far greater savings. About I.7 million Americans contract infections each year in hospitals - almost 100 ooo die - with Medicare paying to treat more than $60 \%$ of them. Catheter-associated urinary tract infections alone cost \$45 I million annually, according to the US Centers for Disease Control and Prevention.

Hospitals themselves will pay for the additional procedures and extended hospital stays required to fix the problems; the regulations expressly forbid them from shifting the costs to patients.

Emanating from a deficit-reduction law passed by the US Congress in 2005, the new rules require hospitals to start reporting secondary diagnoses that are present on admission for patients discharged on or after Oct. I. Almost no US hospitals currently record such data.

Hospitals will not be reimbursed for the extra cost of treating patients who acquired conditions while in hospital.

With the help of experts, Medicare selected conditions that were considered preventable, then sought public comment on the proposed changes in May. Medicare anticipates adding 3 more conditions next year.

Private insurers are expected to follow Medicare's lead, said Susan Pisano,

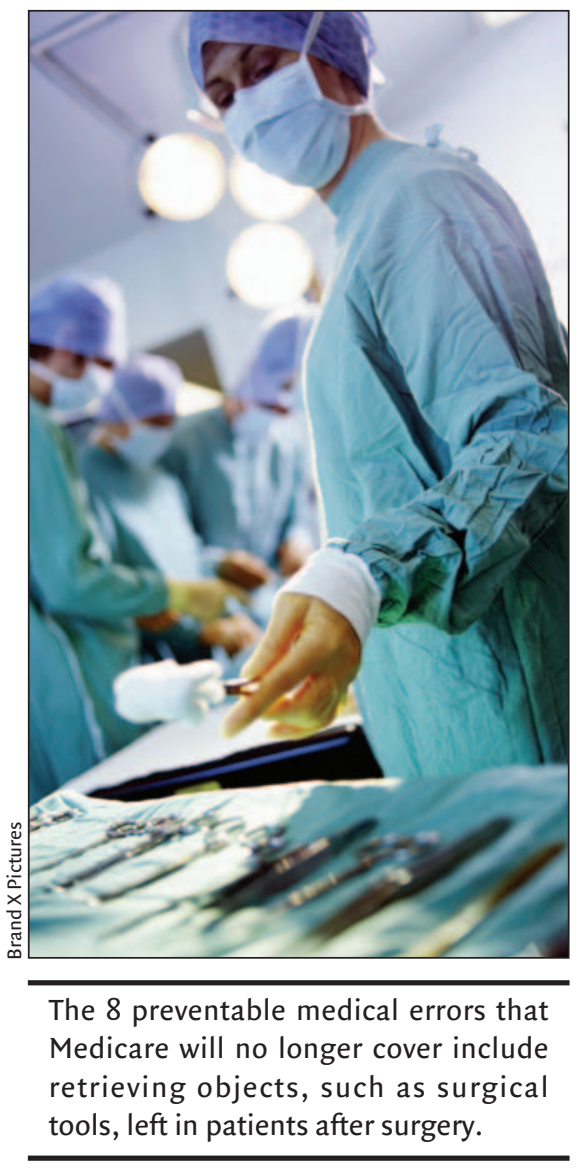

a vice-president of America's Health Insurance Plans, a trade group. A few US insurers already have similar policies, and "a considerable number" are mulling such changes. "Payment incentives play a good role in encouraging quality and enforcing it where it exists," she said.

Nancy Foster, vice-president for quality and patient safety policy for the American Hospital Association, said she couldn't predict whether the changes would increase hospital costs. But when hospitals make clearly preventable errors that cause harm to patients, they shouldn't expect Medicare to pay for them, she said.

Other conditions, such as falls, are not completely preventable, Foster said. "We have lots of ways of reducing falls, but nothing approximating zero yet."

The new reporting requirements are likely to "significantly" increase laboratory testing to determine if infections are present on admission, Foster said. But how, she asked, do physicians admitting patients distin- guish between a preliminary bedsore and mere redness of skin? Hospital are also inventing data collection systems on the fly, she said. "You need to lay the groundwork, if you're going to make this a national policy."

The American Medical Association declined to comment on the new rules, saying it had not yet reviewed them. In June, the association told Medicare that cutting payments to hospitals for treating complications that are "often a biological inevitability regardless of safe practice" was discriminatory. The proposed changes could result in delay or denial of care to vulnerable patients, including elderly patients with compromised immune systems, the association added.

"Continued access to care for these patients has already become more difficult due to the costs of care and this policy could significantly compound the problem by leading hospitals to erect barriers to admission," it wrote.

Consumer groups generally lauded the changes. But the founder of the Committee to Reduce Infection Deaths, a non-profit patient advocacy group, decried the decision to strike staphylococcal aureus septicemia infections from the final list. Betsy McCaughey said Medicare's historical indifference to quality has increased costs and put patients at risk. "Hospitals aren't going to clean up until their biggest customer in this country demands it," she said.

The new policy also drew a favourable response from heavy hitters like the New York Times, which, in an editorial, pointed to the "perversity" of a system that rewards incompetence by paying hospitals more when surgeries are complicated by infection caused by the hospitals' own sloppiness.

Medicare should also consider docking the doctors who make the mistakes, the Times argued. Ken Connor of the Center for a Just Society, a conservative Christian think tank, went even further, urging Bush to require hospitals to repay the "billions of dollars" they have received from Medicare over the decades to fix their mistakes. - Janet Brooks, Salt Lake City, Utah

DOI:Io.1503/cmaj.071347 